\title{
The potential of human skin and plant-based odours combination to attract malaria mosquito, Anopheles gambiae s.s. in Laboratory and Semi field settings.
}

ELISON ELIEZA KEMIBALA ( $\square$ elisonmwebembezi@gmail.com )

University of Dar es Salaam

Agenor Mafra-Neto

ISCA Technologies Inc

Teun Dekker

Universitetet i Oslo

Jesse Saroli

ISCA Technologies Inc

Rodrigo Silva

ISCA Technologies Inc

Anitha Philbert

University of Dar es Salaam

Kija Ng'habi

University of Dar es Salaam

Leonard E.G. Mboera

Sokoine University of Agriculture

Research

Keywords: Anopheles gambiae, mosquito, attraction, Vectrax, human skin, odour

Posted Date: January 28th, 2020

DOI: https://doi.org/10.21203/rs.2.22062/v1

License: (1) (1) This work is licensed under a Creative Commons Attribution 4.0 International License.

Read Full License 


\section{Abstract}

Background Knowledge on mosquito attraction to various host-mimicking odors provides a potential opportunity for designing effective tools for surveillance and control of potential disease vectors. This study was carried out to explore the potential of combining plant and human mimicking odours to attract Anopheles gambiae s. s . in the laboratory and semi field settings in Tanzania. Methodology Blood fed and unfed female Anopheles gambiae s.s. mosquitoes were offered choices between human skin, plantbased odours or combination thereof in laboratory and semi-field setting. The captured mosquitoes from each source were scored for comparison. Results In laboratory and semi-field trials, the tested attractants combination of plant and human-mimicking odours found to be slightly attractive (approximately $10 \%$ higher) than either individual attractant to blood fed female mosquitoes under lab and semi-field settings. The unfed female mosquitoes were more attracted to Vectrax (plant-based materials) by more than $10 \%$ in comparison to human skin-based attractant both in lab and semi-field settings. In semi-field settings particularly, more unfed female mosquitoes were attracted to Vectrax than to the combination $(p<0.01)$. Conclusions The findings that the combination blend (human host and plant based) semiochemical increases mosquito attraction hold the potential to increase efficiency of mosquitoes trapping devices in both indoor and outdoor settings. This may provide valuable tools for mosquito vector management programs. Moreover, these results also suggest the potential of the combination odours to be used in the attract and kill strategies.

\section{Introduction}

There has been a decline of malaria incidence globally during the past decade [1-5]. Several interventions have been reported to play substantial roles in this decline, including improved case management and malaria diagnostic methods, as well as the deployment of Long lasting insecticidal treated nets (LLIN) and indoor residual spraying (IRS) to reduce interactions between mosquito vectors and people $[6,7,2,8]$. However, the positive results achieved through the universal coverage of LLINs and IRS have enticed the community, policy makers, and other malaria control stakeholders to direct their efforts on these interventions while giving less attention to other alternative vector control techniques [9]. The historical background of vector control campaigns, particularly those seeking to reduce transmission of malaria, has shown that over-reliance on a single approach or a certain group of insecticides leaves the campaign vulnerable to failure due to the development of resistance [10].

Repeated application of insecticides of the same mode of action can lead to selection pressure on targeted mosquitoes' species' such that selected mosquitoes with survival advantages cannot be controlled by the same insecticide or an insecticide with similar mode of action and hence failure of the intervention[10]. This setback was revealed in the first global malaria eradication campaign between 1955 and 1969, which was intensively based on indoor residual spraying of dichlorodiphenyltrichloroethane (DDT) of which on long run mosquito developed resistance against it hence failure of the campaign in different areas $[11,12,13,9]$. More recently, mosquito resistance to pyrethroid-based insecticides used for malaria vector control has been reported in several countries [14, 
$9,15,16]$. In the face of increasing mosquito resistance and a threat of malaria resurgence, the World Health Organization recommends the use of integrated vector management (IVM) [17]. The integrated Vectors management recommended by WHO demands all intervention to utilize several scientific proved methods in the move to control all vectors [17]. Hence to compliment current interventions and sustain the achievements gained through universal coverage of LLINs and IRS, further exploration of novel and innovative strategies is of paramount importance.

Despite their success, LLINs and IRS approaches involve no host odour attraction. Therefore, attract and kill is one of the novel techniques/ approaches that is currently attracting attention among scientists [18]. The attract-and-kill strategy is an innovative technique to control diseases vectors and other insect pests involving combining of an attracting agent such as extract of flower odours or human skin mimic odours and a killing agent such a potent insecticide [18]. The use of host odour-based attractants technique has great potential in that, it is much more specific to the target insect and has less negative impact on the environment. Similarly, other control techniques that involve the use of Behaviour-modifying chemicals (semio-chemicals). The use of this technique is based on the concept of interaction between mosquitoes and an attractive source. These approaches can be employed in several ways to control insect pests or disease vectors. For example, semiochemicals can interfere with insect mating or insect oviposition $[19,20]$. Also, volatile semio-chemicals emitted by a source can mimic odours of a potential host for a blood meal, a supply of nutritive, sugar-rich nectar, or a suitable oviposition site [21Therefore, the development of approaches such as toxic baits using these attractive semiochemicals has the potential to contribute to mosquito management and control of mosquito-borne infections [22].

The attraction of mosquitoes to single compounds has been demonstrated in mosquitoes' vectors by several studies [23-25]. However, recently, there have been attempts to study the additive or synergistic effects of blends of semiochemicals to maximize their potency as mosquito attractants [26-29]. The objective of this study was to determine the effect of blends of human skin-based odours (material containing lactic acid and other human skin mimic compounds) and plant-based odours (containing a potent blend of floral attractants) in attracting the female malaria mosquito, Anopheles gambiae s.s.

\section{Material And Methods}

\section{Study area}

This study was carried out in Muheza District located in the northeast of Tanzania $\left(5^{\circ} 13^{\prime} \mathrm{S}, 38^{\circ} 39^{\prime} \mathrm{E}\right.$; altitude $193 \mathrm{~m}$ ). The district is characterized by a humid and warm climate almost throughout the year. The average annual rainfall in Muheza is $1,000 \mathrm{~mm}$ with two seasonal peaks i.e. a main peak between March and May, and a less pronounced one between November and December. The mean temperature in the area is $26^{\circ} \mathrm{C}$. The cooler months are between June and September while the warmer ones are between October and May. The experiments were carried out in an insectary and in mosquito spheres (semi-field environment) at Amani Research Centre of the National Institute for Medical Research. 


\section{Rearing mosquitoes for experiments}

Anopheles gambiae s.s Kisumu strain, brought from Kenya Medical Research Institute (KEMR) were colonized and maintained in a controlled environment $\left[27 \pm 1^{\circ} \mathrm{C}, 65 \pm 5 \%\right.$ relative humidity $(\mathrm{RH})$, and at a 12 h:12 h light: dark cycle] at the Amani Research Centre since early 1982. Anopheles larvae were reared in plastic trays $(20 \mathrm{~cm} \times 30 \mathrm{~cm} \times 10 \mathrm{~cm})$ filled with distilled water in groups of 250 per tray and fed on fish food $\left(\right.$ Tetramin $\left.^{\mathrm{R}}\right)$ once a day. Adults were kept in cages $(30 \mathrm{~cm} \times 30 \mathrm{~cm} \times 30 \mathrm{~cm})$ with access to a $10 \%$ sucrose solution for sustenance. To enable reproduction, female mosquitoes were blood-fed on rabbits according to Standard Operating Procedures (SOPs) approved by the Tanzania Medical Research Coordinating Committee [30]. European Community guidelines and standards were followed in rabbit rearing [30]. Only female mosquitoes were used for both laboratory and semi-field trials.

\section{Chemical/ Odour blend used}

\section{a) Vectrax}

Vectrax is sprayable liquid formulation that mimics natural mosquito's semio-chemical odors, containing a potent blend of floral attractants and sugar-based feeding stimulants [18,31].

b) Skin lure

The Skin Lure is a matrix material containing human skin mimic compounds formulated in SPLAT (materials that allow slow release of odour). The product is a non-commercial produced at ISCA technologies company is US and supplied in bubble caps form.

\section{Experiment procedures}

Laboratory experiments: All experiments were conducted in the insectary in a controlled environment $\left[27 \pm 1^{\circ} \mathrm{C}, 65 \pm 5 \%\right.$ relative humidity $(\mathrm{RH})$, and at a $12 \mathrm{~h}: 12 \mathrm{~h}$ light: dark cycle]. Mosquitoes were released into rectangular mesh cages ( $91 \mathrm{~cm}$ length, $46 \mathrm{~cm}$ width and $30 \mathrm{~cm}$ height) 20 mosquitoes per cage in which tested substrate choices were offered (Figure 1).

In the first experiments, the attractiveness of both, Vectrax and skin-lure attractants was compared to each other on blood fed and unfed female Anopheles mosquitoes. Then attractiveness of each attractant, Vectrax and skin- lure was compared against the combination of the two in separate cages on blood fed and unfed female Anopheles gambiae mosquitoes.

Each attractant was offered in a black plastic bowl (10 cm length $\times 5 \mathrm{~cm}$ width and $4 \mathrm{~cm}$ height) with a lid. The bowl had four evenly spaced windows $(2 \mathrm{~cm} \times 5 \mathrm{~cm})$ into the walls and the fifth one on the lid, it was through these windows mosquitoes had access to the inside of the bowl. A blue panel with sticky trap was fitted onto the bottom of the black plastic bowl to trap the entered mosquitoes (Figure 2). A petri dish with vectrax were placed at the bottom of the trap on sticky trap panel 
The attractant (Skin lure bubble cap) were hanged through the window on the lid of the bowl. For the odour combination, both two attractants were placed in the same bowl, while skin lure releasing bubble cap hanged and petri dish with vectrax placed at the bottom of the bowl. After the attractant treatments were placed in the bowl traps, the bowls with compared choices were placed on opposing corners of each mesh screen about $61 \mathrm{~cm}$ apart to reduce the mixing up of odours (Figure 1).

Adult female Anopheles mosquitoes, starved for two hours before the experiment, were released into 91 $\mathrm{cm}$ mesh screen cages 30 minutes prior to placing traps in the cage, in order to allow for acclimation. After 24 hours, the number of mosquitoes caught in each bowl trap was counted and recorded. Four replicates of each experiment trial to test attractiveness of each attractant were performed.

\section{Semi-field experiments}

These experiments were conducted in mosquito-spheres [32] over a period of 30 days. Under this setting, blood fed or unfed female mosquitoes, were simultaneously offered four treatments: the skin lures, Vectrax, a combination of the two, and a control (no attractant). In this semi-field setting, each treatment was offered using a specially designed apparatus called a mini-zumba trap (Figure 4). Mini-zumba traps uses a fan to draw in mosquitoes from the opening on the top of the trap into a catch bag inside. The lure is placed in the bottom of the trap, outside the bag, and air that is drawn in by the fan passes over the lure and disperses the odour. The scented air then travels up the walls of the trap and is ejected horizontally out of the baffles (holes) on the sides of the lid. This ensures that odour of the lure gets spread away from the trap to draw the mosquitoes in. Each Mini-Zumba trap was labelled according to its content.

The four Mini-Zumba traps were place in each of the four corners of the mosquito sphere, approximately 7 meters from each other. In each experiment, a total of 200 adult female Anopheles mosquitoes (starved for 2 hours) were released in the mosquito-sphere at 18:00 hr. The traps were retrieved the following morning at 06:00 hr. Mosquitoes captured in each trap were collected counted and recorded. Mosquito collection proceeded for 2 days consecutively with no new mosquitoes being released. A buffer of 1 day was maintained for cleaning and allowing the sphere to get aired out, emptied and cleaning of miniZumba traps. Also, this allowed uncaptured mosquitoes to die before the next experiment. Five experimental replicates were made for both blood fed and unfed mosquitoes experiments following same procedures described above. In each experimental replicate, attractants were shifted position to correct for positional bias hence a total of ten (10) replicates, five (5) for unfed and five (5) for fed.

\section{Data analysis}

The number of mosquitoes captured by the presented attractant in each treatment were summarized in Microsoft Excel and then transferred to Stata software (StataCorp. 2013) for analysis.

\section{Ethical considerations}

The study was approved by the Medical Research Coordinating Committee of the National Institute for Medical Research, Tanzania (Research Permit Ref. No. NIMR/HQ/R.8a/Vol.IX/1584). 


\section{Results}

\section{Laboratory experiments}

In the laboratory experiment, a total 480 female mosquitoes (blood fed $=240$; unfed $=240$ ) were released during the experiment. A total of 292 females were captured (157 unfed females and 135 blood fed females).

The total number of blood fed female mosquitoes attracted to the combination of attractants was slightly higher compared to those attracted to individual attractants; skin lure $(P>0.01)$ and vectrax $(P>0.01)$, but the difference was not statistically significant. There was no significant difference in attraction to individual attractant (skin lure and vectrax) for both blood fed $(P=0.6910)$ and unfed $(P=0.3593)$ female mosquitoes. For unfed female mosquitoes the combination of Skinlure and vectrax attracted significantly higher number of mosquitoes in comparison with the attraction of skin lure alone $(P=0.0204)$. However, when the combination of odours were compared to vectrax alone the difference was not statistically significant $(P=0.1194)$. Therefore under laboratory settings, it was observed that combination of odours (Skin lure and Vectrax) was more effective in attracting females than when skin lure alone was used $(P=0.02)$, (Table 1$)$

Table 1: The average number of mosquitoes captured in Skinlure, Vectrax, and combination of the two traps under laboratory settings

\begin{tabular}{|c|c|c|c|c|c|}
\hline \multirow{2}{*}{$\begin{array}{l}\text { Attractants/blood feeding } \\
\text { status }\end{array}$} & \multirow{2}{*}{$\begin{array}{l}\text { Total mosquitoes } \\
\text { captured }\end{array}$} & \multirow{2}{*}{$\begin{array}{l}\text { Percent } \\
\text { captured }\end{array}$} & \multicolumn{2}{|c|}{$95 \%$ C. I. } & \multirow{2}{*}{$\begin{array}{l}\text { P- } \\
\text { value }\end{array}$} \\
\hline & & & Lower & Upper & \\
\hline \multicolumn{5}{|l|}{ Blood fed mosquitoes } & \multirow{3}{*}{0.5952} \\
\hline SKINLURE & 44 & 27.50 & 14.73 & 41.27 & \\
\hline SKINLURE+VECTRAX & 53 & 33.13 & 20.34 & 45.66 & \\
\hline SKINLURE+VECTRAX & 53 & 33.13 & 20.34 & 45.66 & \multirow[t]{2}{*}{0.3520} \\
\hline VECTRAX & 38 & 23.75 & 10.42 & 37.58 & \\
\hline SKINLURE & 44 & 27.50 & 14.73 & 41.27 & \multirow[t]{2}{*}{0.6910} \\
\hline VECTRAX & 38 & 23.75 & 10.42 & 37.58 & \\
\hline \multicolumn{5}{|l|}{ Unfed mosquitoes } & \multirow{3}{*}{0.0204} \\
\hline SKINLURE & 35 & 21.88 & 8.28 & 35.72 & \\
\hline SKINLURE+VECTRAX & 72 & 45.00 & 33.51 & 56.49 & \\
\hline SKINLURE+VECTRAX & 72 & 45.00 & 33.51 & 56.49 & \multirow[t]{2}{*}{0.1194} \\
\hline VECTRAX & 50 & 31.25 & 18.18 & 43.82 & \\
\hline SKINLURE & 35 & 21.88 & 8.28 & 35.72 & \multirow[t]{2}{*}{0.3593} \\
\hline VECTRAX & 50 & 31.25 & 18.18 & 43.82 & \\
\hline
\end{tabular}

\section{Semi-field experiments}

In the mosquito sphere trial, a total of 2,000 female mosquitoes (blood fed $=1,000$; unfed $=1,000$ ) were released. Of the released mosquitoes, $259(25.9 \%)$ of unfed mosquitoes and $338(33.8 \%)$ of fed 
mosquitoes were trapped.

The Vectrax trap was more effective in attracting unfed female mosquitoes $(n=119)$ when compared to a combination of odour (skin lure and Vectrax $(n=74),(p<0.01)$ or skin lure alone $(p<0.01)$. Blood fed mosquitoes trapped in the combination of odour did not differ significantly from those trapped in vectrax alone $(P=0.1759)$ or skin lure alone $(0.2548)$ (Table 2$)$.

Table 2: Average attractions to different attractants in mosquito spheres

\begin{tabular}{|c|c|c|c|c|c|c|}
\hline \multirow{2}{*}{$\begin{array}{l}\text { Attractants/Abdominal } \\
\text { Status }\end{array}$} & \multirow{2}{*}{$\begin{array}{l}\text { Total } \\
\text { trapped }\end{array}$} & \multirow[t]{2}{*}{ mosquitoes } & \multirow{2}{*}{$\begin{array}{l}\text { Percent } \\
\text { trapped }\end{array}$} & \multicolumn{2}{|c|}{$95 \%$ C. I. } & \multirow{2}{*}{$\begin{array}{l}\text { P- } \\
\text { value }\end{array}$} \\
\hline & & & & Lower & Upper & \\
\hline \multicolumn{7}{|l|}{ Blood fed mosquitoes } \\
\hline VECTRAX & 103 & & 51.50 & 41.85 & 61.15 & \multirow[t]{2}{*}{0.1759} \\
\hline VECTRAX+SKINLURE & 121 & & 60.50 & 51.79 & 69.21 & \\
\hline CONTROL & 8 & & 4.00 & -9.58 & 17.58 & \multirow[t]{2}{*}{0.0096} \\
\hline VECTRAX & 103 & & 51.50 & 41.85 & 61.15 & \\
\hline CONTROL & 8 & & 4.00 & -9.58 & 17.58 & \multirow[t]{2}{*}{0.0018} \\
\hline VECTRAX+SKINLURE & 121 & & 60.50 & 51.79 & 69.21 & \\
\hline SKINLURE & 106 & & 53.00 & 43.50 & 62.50 & \multirow[t]{2}{*}{0.8282} \\
\hline VECTRAX & 103 & & 51.50 & 41.85 & 61.15 & \\
\hline SKIN LURE & 106 & & 53.00 & 43.50 & 62.50 & \multirow[t]{2}{*}{0.2548} \\
\hline VECTRAX+SKINLURE & 121 & & 60.50 & 51.79 & 69.21 & \\
\hline CONTROL & 8 & & 4.00 & -9.58 & 17.58 & \multirow[t]{2}{*}{0.0075} \\
\hline SKINLURE & 106 & & 53.00 & 43.50 & 62.50 & \\
\hline \multicolumn{7}{|l|}{ Unfed mosquitoes } \\
\hline VECTRAX & 119 & & 59.50 & 50.68 & 68.32 & \multirow[t]{2}{*}{0.0024} \\
\hline VECTRAX+SKINLURE & 74 & & 37.00 & 26.00 & 48.00 & \\
\hline CONTROL & 10 & & 5.00 & -8.51 & 18.51 & \multirow[t]{2}{*}{0.0009} \\
\hline VECTRAX & 119 & & 59.50 & 50.68 & 68.32 & \\
\hline CONTROL & 10 & & 5.00 & -8.51 & 18.51 & \multirow[t]{2}{*}{0.0437} \\
\hline VECTRAX+SKINLURE & 74 & & 37.00 & 26.00 & 48.00 & \\
\hline SKINLURE & 56 & & 28.00 & 16.24 & 39.76 & \multirow[t]{2}{*}{0.0001} \\
\hline VECTRAX & 119 & & 59.50 & 50.68 & 68.32 & \\
\hline SKINLURE & 56 & & 28.00 & 16.24 & 39.76 & \multirow[t]{2}{*}{0.2803} \\
\hline VECTRAX+SKINLURE & 74 & & 37.00 & 26.00 & 48.00 & \\
\hline CONTROL & 10 & & 5.00 & -8.51 & 18.51 & \multirow[t]{2}{*}{0.1194} \\
\hline SKINLURE & 56 & & 28.00 & 16.24 & 39.76 & \\
\hline
\end{tabular}

\section{Discussion}

The results of both the laboratory and semi-field studies reported here demonstrate increased attraction of female An. gambiae s.s. mosquitoes (fed and unfed) to a combination of vectrax (a plant-based 
semiochemical) lure and skin lure (a human skin-mimicking) lure. The combination of two lures, each representing an important food diet required by female mosquitoes throughout their lives. The Vectrax a plant based semiochemicals is important for female mosquitoes as a source of sugar for energetic purpose [33]. The skin lures a human mimicking semiochemicals is important to female mosquitoes as a source of blood meal for enrichment of eggs [23]. In the Laboratory settings particularly, the combination of the two attractants enticed much of the population to the designated point in comparison to individual attractants.

The attraction of the two semiochemicals used in this study, has been demonstrated by a number studies; A study of female Aedes aegypti demonstrated higher affinity to the blend of $1 \%$ L-lactic and 1Octen-3-ol (human skin odour based compound) when compared to individual fruit -based toxic sugar bait [34]. Over the past sixty years, attraction studies have put forth a body of evidence on the attraction of female mosquitoes to blood host mimicking odours and plant-based attractants, although these attractants have been studied separately [35-42].

The affinity of a female mosquito to a particular odour or food source depends on its physiological status, e.g. blood fed/unfed, mated or gravid [43,33]. For example, female Anopheles gambiae s.s mosquitoes, aged between 5 - 14 days, are much attracted to lactic acid, a human host- signifying odour, under olfactometer trial in the lab [23]. This preference is different to that of newly emerged mosquitoes that prefers feeding on sugar solutions first before seeking a host's blood source [33]. Studies have also shown that, older mosquitoes that had undergone several gonotrophic cycles were more attracted to sugar sources in the field settings]44].

In this study, unfed female mosquitoes An. gambiae s.s., were much more attracted to the Vectrax (plantbased attractant) than to skin lure in semi field setting $(p<0.01)$. This demonstrates a well-known mosquito feeding habit: Most female mosquitoes will take sugar meals before they will seek a blood meal, and some strongly prefer sugar over blood or rarely bite until after a sugar meal or until after several weeks of sugar feeding [33]. Similarly, unfed mosquitoes both in the laboratory and semi-field setting were attracted to the combination of attractants when compared to skin lure alone, indicating that still females were able to detect plant source even when combined to human mimicking odour. However, when tested in the laboratory, the attraction of female mosquitoes to vectrax and skin lure was not statistically significant for both fed and unfed females. The possible explanations to explain why female mosquitoes had higher affinity to Vectrax alone and combination of Vectrax and skin lure, could be due the fact that, females used in this experiment were young females (4-5 days old). There it is possible that females were attracted to plant based semiochemical based on their physiological status, as young females are attracted to sugar source in comparing to older one aged above 5 days [23]. Furthermore, our finding that Vectrax and the combination of Vectrax and skin lure are potential attractant for both young unfed females and blood fed female mosquitoes is supported by other findings that mosquitoes that had first blood meal alternate blood and sugar meal frequently [33]. 
These finding have several potentials for malaria vector control. Blood fed mosquitoes carry the potential for disease parasite transmission [23], Thus being highly attracted to our new odour blends provides an opportunity to target females with of particular physiological status. Also, the higher affinity of unfed females again provides an important opportunity to target young females before they can start seeking blood meal. New vector control approaches can be designed to integrate new odour blends in approaches that focus on attract and kill principles.

Although there was significant attraction of the combination of two odours to female mosquitoes both fed and unfed particularly in the laboratory settings, the study did not compare the attraction of the blends to human. In field settings the bled will be competing with the human being which is the preferred host for malaria vectors. Thus, further investing is needed to reveal the affinity of the new odour blends compared to that of human in natural field settings.

\section{Conclusion}

Blood fed mosquitoes are the only disease transmitters, since it is a group with a chance of carrying pathogens. The increased attraction of this group to the tested blend of attractants provides evidence that the combined substrate has great potential to improve trapping efficacy of infected mosquitoes, thereby giving an accurate picture of mosquitoes' infectivity and the risk of disease transmission. The findings of this study also provide prospects for designing an effective attract and kill system that is more specific to the targeted mosquitoes and more environmentally friendly. This study has shown that the blend of human skin odours and Vectrax increases the mosquito attraction particularly the blood fed ones with potential higher risk of disease transmission and holds a promising future improving mosquito surveillance and control strategies.

\section{Abbreviations}

Cl: confidence interval; ITN: insecticide-treated nets; NIMR: National Institute for Medical Research; WHO: World Health Organization.

\section{Declarations}

The authors declare that they have no competing interests

\section{Ethics approval and consent to participate}

The study was approved by the Medical Research Coordinating Committee of the National Institute for Medical Research (Ref. No. NIMR/HQ/R.8a/Vol.IX/1584). Mosquitoes used in the experiments were raised in an insectary and free from infections. Human volunteers provided an informed consent before participating in the experiments.

Consent for publication: Not applicable. 
Availability of data and material: All available data are included in this article.

Competing interests: All authors declare no conflict of interest.

Funding: This study received financial assistance from Bill and Melinda Gates Foundation through ISCA Technologies, Inc.

\section{Authors' contributions}

LEGM, TD, AM, EEK conceived the idea and designed the study. TD, AP, EEK carried the statistical analysis. LEGM, TD, AM, JS, RS carried out and supervised the experiments. EEK, LEGM wrote the initial draft of the manuscript. All authors read and approved the final version of the manuscript.

\section{Acknowledgements}

We are grateful to Amani Research Centre Management for their permission to utilize the Centre's mosquito spheres and supply of laboratory-reared mosquitoes. We would also like to thank Rukia Ahmed, Mathew Barabara for their field assistance and rearing of mosquitoes. This study received financial assistance from Bill and Melinda Gates Foundation through ISCA Technologies.

\section{References}

1. Ceesay, S.J., Casals-Pascual, C., Erskine, J., Anya, S.E., Duah, N.O., Fulford, A.J.C., Sesa, S.S.S., Abubakar, I., Dunyo,S., Sey, O., Palmer, A., Fofana, M., Corrah, T., Bojang, K.A., Whittle, H.C., Greenwood, B.M., Conway, D.J. Changes in malaria indices between 1999 and 2007 in The Gambia: a retrospective analysis.2008: Lancet 372:1545-1554.

2. O'Meara, W.P., Mangeni, J.N., Steketee, R., Greenwood, B. Changes in the burden of malaria in subSaharan Africa. 2010: Lancet Infect Dis 10:545-555.

3. Steketee, R.W., Campbell, C.C. Impact of national malaria control scale-up programmes in Africa: magnitude and attribution of effects. 2010: Malar J 9:299.

4. Pigott, D.M., Atun, R., Moyes, C.L, Simon, I.H., Peter, W.G. Funding for malaria control 2006-2010: A comprehensive global assessment. 2012 : Malar J 11, 246 (2012) doi:10.1186/1475-2875-11-246.

5. Murray, C.J.L., Rosenfeld, L.C., Lim, S.S., Andrews, K.G., Foreman, K.J., Haring, D., Fullman, N., Naghavi,M., Lozano, R., Lopez, A.D. Global malaria mortality between 1980 and 2010: a systematic analysis. 2012: Lancet 379:413-431.

6. Okiro, E.A., Hay, S.I., Gikandi, P.W., Shahnaaz, K. S., Abdisalan,M. N., Norbert, P., Kevin,M.,Robert,W.S .The decline in paediatric malaria admissions on the coast of Kenya. 2007 : Malar J 6, 151; doi:10.1186/1475-2875-6-151.

7. Otten, M., Aregawi, M., Were, W., Corine, K., Ambachew, M., Worku, B., Daddi, J., Khoti, G., Ryuichi, K., Eline, K., Daniel,L.,Mark, G. Initial evidence of reduction of malaria cases and deaths in Rwanda and 
Ethiopia due to rapid scale-up of malaria prevention and treatment . 2009: Malar J 8, 14, doi:10.1186/1475-2875-8-14.

8. Erin Eckert, L.S., Florey, J.E,. Tongren, S., René Salgado, A.R., Jean Pierre, H. E. H., Kaend M., Noella,U., Monique M., Corine, K. Impact evaluation of Malaria Control Interventions on Morbidity and All-Cause Child Mortality in Rwanda, 2000-2010. 2017 : Am J Trop Med Hyg 97(3 Suppl): 99-110.

9. Russell, T.L., Govella,N.J., Azizi,S., Drakeley,C.J., Kachur,S.P., Killeen,G.F .Increased proportions of outdoor feeding among residual malaria vector populations following increased use of insecticidetreated nets in rural Tanzania. 2012: BioMed Central, Malaria journal, Vol. 10, p. 80.

10. Vincent, C., Raphael,N. Distribution, Mechanisms, Impact and Management of Insecticide Resistance in Malaria Vectors: A Pragmatic Review, Anopheles mosquitoes - New insights into malaria vectors, Sylvie Manguin. s.I. , 2013 : IntechOpen, DOI: 10.5772/56117. Available from: https://www.intechopen.com/books/anopheles-mosquitoes-new-insights-into-malariavectors/distribution-mechanisms-impact-and-management-of-insecticide-resistance-in-malariavectors-a-pragmat.

11. WHO. Action Plan for the Reduction of Reliance on DDT in Disease Vector Control. 2001 : http://whqlibdoc.who.int/hq/2001/WHO_SDE_WSH_01.5.pdf. Accessed 5 Aug 2015.

12. Nauen, R. Perspective Insecticide resistance in disease vectors of public health importance. 2007: Pest Manag Sci 63:628-633.

13. ) José, A.N., Matiana, G., Pedro, L.A. Some Lessons for the Future from the Global Malaria Eradication Programme (1955-1969). 2011 : https://doi.org/10.1371/journal.Plos med.1000412.

14. Ranson, H., N’Guessan, R., Lines, J., Moiroux, N., Nkuni, Z., Corbel, V. Pyrethroid resistance in African anopheline mosquitoes: what are the implications for malaria control?,. 2011 : TRENDS OF PARASITOLOGY ,Volume 27, Issue 2;Pages 91-98.

15. Charles, S. W., Michael, C., Immo, K., Themba, M., Helen, I., Miranda, N., Andrea, R., John, M., Kayla, G. B., Janet, H. Impact of pyrethroid resistance on operational malaria control in Malawi. 2012 : PNAS 109 (47) 19063-19070.

16. Kabula, B. , Tungu, P. , Malima, R. , Rowland, M. , Minja, J. , Wililo, R. , Ramsan, M. , Mcelroy, P. D., Kafuko, J. , Kulkarni, M. , Protopopoff, N. , Magesa, S. , Mosha, F. And Kisinza, W. Distribution and spread of pyrethroid and DDT resistance among the Anopheles gambiae complex in Tanzania. 2014 : Med Vet Entomol, 28: 244-252. doi:10.1111/mve.12036.

17. WHO. Global Strategic Framework for Integrated Vector Management. 2004 : http://www.who.int/malaria/publications/atoz/who_cds_cpe_pvc_2004_10/en/. Accessed 5 Aug 2014.

18. Mafra-Neto, A., Dekker, T. Novel Odor-based Strategies for Integrated Management of Vectors of Disease. Current Opinion in Insect Science. 2019 :https://doi.org/10.1016/j.cois.2019.05.007.

19. Lewis, W.J., Martin, W.R. Semiochemicals for use with parasitoids: Status and future.1990: Journal of Chemical Ecology, Volume 16, Issue 11, pp 3067-3089. 
20. Moraes, M.C.B., Pareja, M., Laumann, R.A., Borges, M. s.The Chemical Volatiles (Semiochemicals) Produced by Neotropical Stink Bugs (Hemiptera: Pentatomidae). 2008: Neotropical Entomology 37(5):489-505.

21. Zwiebel, L.J., Takken, W. Olfactory regulation of mosquito-host interactions. 2004 : Insect Biochem Mol Biol 34:645-52.

22. Allan S. A., Bernier U. R., Kline D. L. Laboratory Evaluation of Avian Odors for Mosquito (Diptera: Culicidae) Attraction. 2006: J Med Entomol, Vol. 43, pp. 226-231.

23. Dekker T, Steib B, Cardé RT, Geier M. L-lactic acid: a human-signifying host cue for the anthropophilic mosquito Anopheles gambiae.2002 : Med Vet Entomol. 16:91-98. doi: 10.1046/j.0269283x.2002.00345.x.

24. Njiru, B.N., Mukabana, W.R., Takken, W., Knols, B.G . Trapping of the malaria vector Anopheles gambiae with odour-baited MM-X traps in semi-field conditions in western Kenya. 2006 : Malar J. 5:39. doi: 10.1186/1475-2875-5-39.

25. Mukabana, W.R., Mweresa, C.K., Otieno, B., Omusula, P., Smallegange, R.C., Van Loon, J.J.A., Takken, W. A novel synthetic odorant blend for trapping of malaria and other African mosquitoes. 2012: J Chem Ecol. 38:235-244. doi: 10.1007/s10886-012-0088-8.

26. Okumu, F.O., Killeen, G.F., Ogoma, S., Biswaro, L., Smallegange, R.C., Mbeyela, E., Titus, E., Munk, C., Ngonyani, H., Takken, W., et al. Development and field evaluation of a synthetic mosquito lure that is more attractive than humans. 2010: PLoS One 5 doi: 10.1371/journal.pone.0008951.

27. MENGER, D. J., VAN LOON, J. J. and TAKKEN, W. Assessing the efficacy of candidate mosquito repellents against the background of an attractive source that mimics a human host. 2014: Med Vet Entomol, 28: 407-413. doi:10.1111/mve.12061.

28. Whitney, A.Q., Günter,C.M., Edita,E.R., Sandra,A.A., Kristopher,L.A.,John,C.B., Michal,L.S., Jodi,M.S., Vasiliy,D.K., et al. Evaluation of attractive toxic sugar bait (ATSB)-Barrier for control of vector and nuisance mosquitoes and its effect on non-target organisms in sub-tropical environments in Florida. 2014: Acta Tropica,Volume 131, Pages 104-110.

29. Van Loon, J.J., Smallegange, R.C., Bukovinszkiné-Kiss, G., Jacobs, F., De Rijk, M., Mukabana, W.R., Verhulst, N.O., Menger, D.J. \& Takken, W Mosquito Attraction: Crucial Role of Carbon Dioxide in Formulation of a Five-Component Blend of Human-Derived Volatiles. 2015 : Journal of Chemical Ecology 41(6): 567-573. doi: 10.1007/s10886-015-0587-5.

30. Schorkopf, D.P., Christos, G.S., Mboera,L.E.G., Mafra-Neto,A., Ignel,R., Dekker.T. Combining Attractants and Larvicides in Biodegradable Matrices for Sustainable Mosquito Vector Control.2016:PLoS Negl Trop Dis 10(10):e0005043. doi:10.1371/journal.pntd.

31. Agenor, M.,Jesse,S.,Rodrigo,O.S., Leonard,E. M., Graham,B.W.,Woodbridge,F., Kim,S.,Babak,E., Daniel,E.S., Thomas,D., Elison,E.K., Rafael,B.,Teun,D. Getting Them Where They Live-SemiochemicalBased Strategies To Address Major Gaps in Vector Control Programs: Vectrax, SPLAT BAC, Trojan Cow, and SPLAT TK. 2018 : Advances in the Biorational Control of Medical and Veterinary Pests Chapter 7pp 101-152. 
32. Knols, B.G., Njiru, B.N., Mathenge, E.M. et al. MalariaSphere: A greenhouse-enclosed simulation of a natural Anopheles gambiae (Diptera: Culicidae) ecosystem in western Kenya. 2002 : Malar J 1, 19; doi:10.1186/1475-2875-1-19.

33. Foster, W.A.Mosquito sugar feeding and reproductive energetics. s.I. : Annu Rev Entomol 40:443-74, 1995.

34. Scott-Fiorenzano, J.M., Fulcher, A.P., Seeger, K.E. et al. Evaluations of dual attractant toxic sugar baits for surveillance and control of Aedes aegypti and Aedes albopictus in Florida. 2017: Parasites Vectors 10, 9; doi:10.1186/s13071-016-1937-Z.

35. Kline, D.L., Takken, W., Wood, J.R., Carlson, D.A. Field studies on the potential of butanone, carbon dioxide, honey extract, 1-octen-3-ol, l-lactic acid and phenols as attractants for mosquitoes. 1990: Med Vet Entomol 4:383-91.

36. Kline, D.L., Bernier, U.R., Hogsette, J.A. Efficacy of three attractant blends tested in combination with carbon dioxide against natural populations of mosquitoes and biting flies at the lower Suwannee Wildlife Refuge. 2012 : J Am Mosq Control Assoc 28:12.

37. Bernier, U.R., Kline, D.L., Posey, K.H., Booth, M.M., Yost, R.A., Barnard, D.R . Synergistic attraction of Aedes aegypti (L.) to binary blends of L-lactic acid and acetone, dichloromethane, or dimethyl disulfide.. 2003: J Med Entomol 40:653-6.

38. Williams, C.R., Bergbauer, R., Geier, M., Kline, D.L., Bernier, U.R., Russell, R.C., Ritchie, S.A. Laboratory and field assessment of some kairomones blends for host seeking Aedes aegypti. 2006 : J Am Mosq Control Assoc 22:641-7.

39. Hoel, D.F., Kline, D.L., Allan, S.A., Grant, A. Evaluation of carbon dioxide, 1-octen- 3-ol, and lactic acid as baits in Mosquito Magnet ${ }^{\mathrm{TM}}$ pro traps for Aedes albopictus in North Central Florida. 2007: Am Mosq Control Assoc 23:11-7.

40. Müller, G.C., Xue, R.D., Schlein, Y .Differential attraction of Aedes albopictus in the field to flowers, fruits, and honeydew. 2011: Acta Trop 118:45-9.

41. El Hadi, M.A.M., Zhang, F.J., Wu, F.F., Zhou, C.H., Tao, J . Advances in fruit aroma volatile research. 2013: Molecules 18:8201-29.

42. Nyasembe, V.O., Tchouassi, D.P., Kirwa, H.K., Foster, W.A., Teal, P.E.A., Borgemeister, C., Torto, B. Development and assessment of plant-based synthetic odor baits for surveillance and control of malaria vectors. 2014 : PLoS One 9(2).

43. Gerberg, E. J., Barnard, D. R. and Ward, R. A. Manual for mosquito rearing and experimental techniques.1994: American Mosquito Control Association, Inc. pp.iv + 98 pp;19952010555.

44. Beier, J.C., Müller, G.C., Gu, W. et al. Attractive toxic sugar bait (ATSB) methods decimate populations of Anopheles malaria vectors in arid environments regardless of the local availability of favoured sugar-source blossoms. 2012 : Malar J 11, 31;doi:10.1186/1475-2875-11-31 